\title{
Experiencia profesional, capital psicológico y engagement. Su relación con el burnout en docentes universitarios
}

\section{Teaching Experience, Psychological Capital and Work Engagement. Their Relationship with the Burnout on University Teachers}

\section{Belén Mesurado*(iD}

Consejo Nacional de Investigaciones Científicas y Técnicas, Buenos Aires, Argentina

Universidad Austral, Buenos Aires, Argentina

ORCID: http://orcid.org/0000-0002-5907-5854

\section{Julieta Laudadío}

Instituto de Investigaciones en Educación de Ciencias Experimentales, Universidad Nacional de

San Juan, Este San Juan, Argentina

ORCID: https://orcid.org/0000-0003-4049-6752

Recibido 21-05-19 Revisado 12-06-19 Aprobado 06-09-19 En línea 11-09-19

*Correspondencia

Email: mesuradob@gmail.com
Citar como:

Mesurado, B., \& Laudadío, J. (2019). Experiencia profesional, capital psicológico y engagement. Su relación con el burnout en docentes universitarios. Propósitos y Representaciones, $\quad 7(3), \quad 12-40$.

http://dx.doi.org/10.20511/pyr2019.v7n3.327 


\section{Resumen}

El presente estudio se plantea dos objetivos el primero es estudiar si las diferentes etapas del desarrollo profesional de docentes hacen variar los niveles de capital psicológico, engagement y burnout en docentes universitarios. Por otro lado, el segundo objetivo es estudiar la relación de las etapas del desarrollo profesional, del capital psicológico y el engagement con el burnout de los docentes universitarios. La muestra es intencional, no probabilística y estuvo compuesta por 250 profesores universitarios, con una media de edad de 39 años $(M=39.5$ y un DT $=11.15)$, $51 \%$ de los participantes eran mujeres. El 38\% de los participantes ejercen la docencia universitaria hace 5 años o menos, el 27\% lo hace entre 6 y 10 años y el 35\% hace más de 11 años que ejerce la docencia universitaria. Para evaluar las variables se utilizaron las versiones españolas del Psychological Capital Questionnaire, Utrecht Work Engagement Scale y el Oldenburg Burnout Inventory. Los resultados indicaron que los docentes más experimentados presentan mayores niveles de capital psicológico (específicamente eficacia, resiliencia y esperanza) y mayor nivel de absorción, una dimensión de engagement, que los menos experimentados. No se encontraron diferencias significativas en los niveles de burnout vinculados a la experiencia académica. Por último, el capital psicológico (específicamente las dimensiones de optimismo y esperanza) así como el engagement (específicamente las dimensiones de vigor y dedicación) inhiben el surgimiento de burnout en docentes universitarios.

Palabras claves: Docencia; Psicología educativa; Efectos psicológicos; estrés.

\section{Summary}

The present study has two objectives. The first one is to study whether the different stages of teacher professional development vary the levels of psychological capital, engagement and burnout on university teachers. On the other hand, the second objective is to study the relationship between the stages of professional development, psychological capital and engagement with the burnout of university teachers. The sample is intentional, not probabilistic and was composed of 250 university professors, with an average age of 39 years $(\mathrm{M}=39.5$ and a $\mathrm{TD}=11.15)$; fifty one per cent of the participants were female. Thirty-eight per cent of the participants have been teaching at the university for five years or less; twenty seven per cent have been doing so for 6 to 10 years, and thirty five per cent have been teaching at the university for more than 11 years. To evaluate the variables, the Spanish versions of the Psychological Capital Questionnaire, Utrecht Work Engagement Scale and the Oldenburg Burnout Inventory were used. The results indicated that more experienced teachers have higher levels of psychological capital (specifically effectiveness, resilience and hope) and higher levels of absorption, an engagement dimension, than less experienced teachers. No significant differences were found in burnout levels linked to academic experience. Finally, psychological capital (specifically the dimensions of optimism and hope) as well as engagement (specifically the dimensions of vigor and dedication) inhibit the emergence of burnout on university teachers.

Keywords: Teaching; Educational Psychology; Psychological Effects; Stress.

\section{Introducción}

La educación superior constituye una base fundamental para la construcción y progreso de la sociedad, de allí el imperativo estratégico de invertir en mejorar la educación en este nivel. Si bien son muchos los desafíos a enfrentar, es patente el rol que cumple el trabajo docente como pieza clave de la mejora. El docente se enfrenta a muchos desafíos vinculados a la preocupación por su formación profesional, a la adquisición de estrategias pedagógicas, a la desmotivación del alumnado, a la falta de recursos, etc. Todos estos desafíos pueden provocar situaciones de estrés y desmotivación en los docentes, que pueden verse agravados o atenuados por el nivel de experiencia docente, así como por sus características individuales. 
De allí que el primer objetivo de este estudio será analizar si existen diferencias en variables personales tales como, el capital psicológico, el engagement y el burnout, de los profesores universitarios de acuerdo con el nivel de experiencia docente de los profesores. El segundo objetivo será analizar la relación entre el nivel de experiencia del profesor universitario, el capital psicológico y el engagement con el burnout laboral.

\section{Experiencia docente}

A la hora de analizar la actividad de los docentes universitarios, no se puede perder de vista la importancia de estudiar los cambios a lo largo de la vida profesional. Ya que el desarrollo profesional de los docentes está atravesado por diferentes etapas: formación inicial, iniciación, estabilización, nuevas preocupaciones, entre otras.

Las investigaciones (Marchesi \& Díaz Fouz, 2007) ponen de relieve que los profesores han de enfrentarse a situaciones muy diversas a lo largo de su vida profesional debido a los cambios en la educación y al estilo de vida de las nuevas generaciones de alumnos, así como al desarrollo y fortalecimiento de sus propias habilidades académicas. Poco a poco el profesor va a acumulando información y experiencia, pero percibe también la dificultad de adaptarse ante las nuevas exigencias educativas. Es por ello que la situación personal de los docentes puede modificarse a lo largo del tiempo bien por transformaciones en el entorno laboral y profesional, bien por cambios en la propia disposición del docente, o, en la mayor parte de los casos en los que esto se produce, por la interacción entre la actitud del profesor y el contexto en el que desempeña su trabajo.

Murillo Torrecilla (2007) a partir de un estudio comparado sobre la carrera profesional y la evaluación docente en 50 países y 55 sistemas educativos en América Latina y Europa, señala la necesidad de formular políticas integrales orientadas a mejorar y sostener la calidad del trabajo docente, como una condición fundamental para asegurar el aprendizaje de los estudiantes. Al indagar acerca de las respuestas que cada sistema educativo da a los mismos interrogantes y desafíos consideran tanto la etapa de formación docente como la carrera profesional para desarrollar, mantener e incrementar la excelencia de los docentes. Entre sus conclusiones, afirma que, junto con criterios de conocimiento de la materia y de competencias de enseñanza, sería necesario tener en cuenta la vocación hacia la docencia. Por ello, parece importante incorporar estrategias que no sólo midan conocimientos y destrezas, también actitudes y compromiso: un buen docente no es aquel que sabe su materia y sabe enseñarla, es, fundamentalmente, un profesional comprometido con su labor y su mejora a lo largo de su carrera docente (Páramo, 2008).

A medida que el docente va adquiriendo mayor experiencia en la enseñanza universitaria va modificando y enriqueciendo no sólo su formación académica, sino que también puede ir modificando características individuales que pueden contribuir o no a atenuar la experiencia de las situaciones de estrés con las que va a enfrentarse.

\section{Capital Psicológico}

Uno de los constructos desarrollados en el marco de la Psicología Positiva es el de Capital Psicológico, definido por Luthans y sus colaboradores como un estado de desarrollo psicológico positivo, que representa la valoración positiva que la persona hace de las circunstancias y su probabilidad de éxito basada en el esfuerzo y la perseverancia (Luthans, Avolio, Avey, \& Norman, 2007). Se trata de un constructo compuesto por cuatro dimensiones: la eficacia, el optimismo, la esperanza y la capacidad de resiliencia. Quiénes poseen un alto nivel de capital psicológico se caracterizan por: (a) tener confianza (eficacia) para asumir y hacer el esfuerzo necesario para tener éxito en tareas desafiantes; (b) haciendo una atribución positiva (optimismo) sobre el éxito ahora y en el futuro; (c) perseverar en los objetivos y, cuando es necesario, redirigir los caminos a los objetivos (esperanza) para tener éxito; y (d) cuando están acosados por 
problemas y adversidades, sostener su esfuerzo (resiliencia) para alcanzar el éxito (Luthans, Youssef, \& Avolio, 2007).

El desarrollo de estos aspectos genera efectos positivos en las instituciones, especialmente en términos de productividad. Los estudios muestran claramente que el capital psicológico se relaciona positivamente con el rendimiento laboral. Las personas marcan la diferencia, sobre todo cuando son autoeficaces, optimistas, esperanzadas y resilientes (Luthans, Youssef, \& Avolio, 2007). Es decir, que son personas capaces de fijar metas personales valiosas, objetivos realistas, crear múltiples maneras para lograr objetivos y para evitar obstáculos. Al mismo tiempo saben identificar la adversidad, reconocer las creencias autodestructivas, darse cuenta de las consecuencias de dichas creencias y cuestionar las creencias contraproducentes. Además de proporcionar apoyo para recuperarse de la adversidad y crecer ante un cambio positivo (Bakker, Rodríguez-Muñoz, \& Derks, 2012).

Recientes estudios, realizados en el ámbito académico, señalaron que las personas con altos niveles de capital psicológico son capaces de establecer objetivos específicos y desafiantes, se encuentran intrínsecamente motivados y más comprometidos con su trabajo y en consecuencia, evidencian un mejor desempeño; tanto en estudiantes (Gutiérrez, Tomás, \& Alberola, 2018; Legé, López Pell, \& Fagnani, 2012) como en docentes (Siu, Bakker, \& Jiang, 2014). En esta misma línea, encontramos investigaciones (Ponce Gutiérrez \& Yáber Oltra, 2012) que analizan el efecto que tiene el capital psicológico sobre el comportamiento organizacional en el ambiente universitario. Entre sus hallazgos señalan la importancia del capital psicológico como un constructo en el que pueden invertir las instituciones para desarrollar sus recursos humanos, ya que propicia actitudes y conductas que favorecen desempeños individuales e instituciones positivas. Los resultados sugieren el valor y el potencial que tiene el capital psicológico, no sólo para la supervivencia de los profesores en ambientes de trabajo más adversos, sino para que puedan prosperar y desarrollar todas sus capacidades.

Es también interesante el aporte de investigaciones que se centran las necesidades psicológicas básicas de los estudiantes de pedagogía en los primeros años de experiencia docente. Entre sus resultados señalan que la satisfacción de la necesidad de competencia, relación, y la autonomía en los estudiantes de pedagogía es considerablemente menor que en los docentes experimentados y la importancia de la experiencia docente para poder incrementar los niveles de satisfacción (Evelein et al., 2008). Al comparar las creencias de efectividad en la enseñanza entre futuros docentes y docentes experimentados también se observan diferencias propias de la experiencia en el aula. Entre las dimensiones analizadas encontramos gestión del aula, disciplina de la clase y eficacia en la enseñanza personal. Los análisis que compararon las expectativas de eficacia mostraron diferencias significativas en la dimensión de gestión y disciplina a favor del grupo de docentes que trabajan. Además, hubo diferencias en la dimensión de gestión y disciplina en cuanto al número de años de experiencia en el grupo de maestros en ejercicio (De la Torre \& Casanova, 2007).

\section{El Engagement docente}

Además del capital psicológico, la literatura refleja también el engagement docente como un novedoso foco de análisis fruto de una aproximación marcada por la psicología positiva (Seligman \& Csikszentmihalyi, 2001).

El concepto de engagement representa un nuevo enfoque que examina las experiencias positivas de los trabajadores y las condiciones favorecedoras de bienestar, y ha sido definido como un estado motivador positivo y persistente relacionado con el trabajo e integrado por las dimensiones de vigor, dedicación y absorción (Salanova, Schaufeli, Llorens Gumbau, Peiró Silla, \& Grau Gumbau, 2000). Así, el componente de vigor se refiere a altos niveles de energía, perseverancia y esfuerzo en la labor que se lleva a cabo a pesar de las dificultades. La dedicación 
hace referencia a niveles elevados de implicación, entusiasmo, inspiración y reto en las tareas ocupacionales. Por último, el factor de absorción se refiere a altos niveles de concentración y de felicidad durante el desempeño de la actividad laboral.

Por otra parte, la psicología positiva presenta el engagement como un constructo motivacional que se relaciona con el trabajo de manera positiva; en donde el individuo experimenta altos niveles de energía y resistencia mental. Las personas con altos niveles de engagement presentan conexiones más energéticas y efectivas con su trabajo, son proactivos, plantean alternativas de mejora, tienen valores que coinciden con los de la institución, y finalmente reciben retroalimentación positiva (Lorente \& Vera, 2010). Se caracterizan por poseer altos niveles de energía, resistencia y activación mental mientras trabajan y están dispuestas a invertir esfuerzo en el trabajo que están realizando, incluso cuando se presentan dificultades en el proceso. Son personas con una alta implicación laboral, junto con la manifestación de un sentimiento de significación, de importancia y desafío frente al trabajo. Al realizar el trabajo experimentan fuertes dosis de concentración y disfrute, que les lleva a percibir que el tiempo pasa rápidamente y que tienen dificultades para desconectarse de lo que están haciendo (Lozano-Paz $\&$ Reyes-Bossio, 2017).

La literatura presenta el síndrome del agotamiento laboral o burnout como la contrapartida del engagement. Conceptualización que se abordará en el siguiente apartado.

\section{El burnout en docentes}

El estrés y la desmotivación docente son problemáticas cada vez más frecuentes en el ámbito educativo. Siguiendo el modelo de Maslach y Jackson (1981), los tres síntomas característicos del agotamiento laboral o burnout son: la baja realización personal, el agotamiento físico y emocional y finalmente la despersonalización. En cuanto a la baja realización personal la persona se siente incapaz de llevar a cabo su trabajo, percibe un rendimiento insatisfactorio que conlleva el desarrollo de sentimientos de fracaso personal, incompetencia y baja autoestima. Otro de los síntomas hace referencia al agotamiento físico y emocional, vivencia de encontrarse agotado emocionalmente, vacío y sin energía la cual aparece asociada con diversas manifestaciones físicas. Y por último la despersonalización, en donde la persona manifiesta actitudes negativas de aislamiento y distanciamiento en relación con las personas para las que trabaja. Esta teoría fue operacionalizada a través del Maslach Burnout Inventory - MBI (Maslach y Jackson, 1981). Si bien esta prueba es una de la más utilizada para la evaluación de burnout presenta la limitación que fue desarrollada exclusivamente para ser usada en profesiones vinculadas a servicios humanos. Posteriormente, se realizó una adaptación de la escala a otras profesiones que se denominó Maslach Burnout Inventory - General Survey (Schaufeli, Leiter, Maslach y Jackson, 1996), sin embargo, no obtuvo tal buenos resultados psicométricos como la escala original. De allí que Demerouti y colegas desarrollaran OLdenburg Burnout Inventory (OLBI), el cual postula la existencia de dos factores agotamiento y desconexión del trabajo (Demerouti, 1999; Demerouti \& Nachreiner, 1998). OLBI propone no sólo evaluar el aspecto afectivo del agotamiento como lo hacer el MBI sino que también agrega la evaluación de los aspectos cognitivos y físicos del agotamiento. Esto facilita la aplicación del instrumento a aquellos trabajadores que realizan trabajo físico, y aquellos cuyo trabajo se trata principalmente de trabajos intelectuales (Demerouti \& Bakker, 2008). Por otro lado, la desconexión en el OLBI se refiere a distanciarse del trabajo en general, del objeto de trabajo y del contenido del trabajo (por ejemplo, falta de interés, así como desagrado por el trabajo). Además, los elementos de desconexión se refieren a la relación entre los empleados y sus trabajos, particularmente con respecto a la identificación con el trabajo y la voluntad de continuar en la misma ocupación (Demerouti \& Bakker, 2008).

En el caso de los profesores, el burnout se puede manifiestar en su vínculo con los alumnos adoptando una relación distante y de escasa receptividad hacia sus demandas. En el origen de esta problemática se encuentra una cierta crisis de identidad profesional y un déficit motivacional del profesor hacia su actividad profesional. Es claro que la profesión docente ha sufrido grandes transformaciones, por los cambios del contexto sociocultural donde el actual 
profesor universitario tiene que llevar a cabo su labor. El docente de hoy en día no solo tiene que responder a sus funciones y responsabilidades investigativo-administrativas, como miembro de una comunidad universitaria, sino que también debe responder a su deber intrínseco de educar los futuros profesionales (Silvero Miramón, 2007). Todos estos hechos generan afectaciones a nivel físico y mental que se traducen en posteriores repercusiones en el ámbito laboral como ausentismo, altos niveles de rotación, bajos niveles de productividad, causados por el llamado síndrome de burnout. Entre las principales características del trabajo que propician el desarrollo del síndrome de burnout en los docentes universitarios se encuentran: multiplicidad de tareas, sobrecarga de trabajo, limitaciones del tiempo para cumplir con las actividades académicas, falta de reconocimiento, carga mental, espacios de trabajo inadecuados, falta de una remuneración que compense los esfuerzos y bajas relaciones sociales en el trabajo (Botero Álvarez, 2012).

El burnout puede considerarse una enfermedad propia de las profesiones asistenciales y especialmente de la docencia caracterizadas por el contacto con personas, ya que a las exigencias laborales se suma la carga de una extrema responsabilidad por el bienestar de otros (Dierendock, Buunk, \& Schaufeli, 2001a). En el centro del fenómeno de burnout está la percepción de la desproporción entre las demandas que el contexto exige y las respuestas que uno es capaz de ofrecer (González Torres, 2003). Se habla de los profesores como un grupo de alto riesgo y son muchos los trabajos que en los últimos años se han dedicado a estudiar el síndrome burnout en los profesores (Darrigrande \& Durán, 2012; Dierendock, Schaufeli, \& Buunk, 2001b; Silvero Miramón, 2007).

De todos modos, los contextos de trabajo varían, al igual que varían las actividades que desempeña cada profesional en su labor, por ello es importante que las instituciones educativas identifiquen y evalúen en su contexto particular, tanto los factores de riesgo psicosocial, como los factores protectores de los docentes para que de esta forma se prevenga o contrarreste el impacto del burnout (Botero Álvarez, 2012). En esta misma línea encontramos estudios más recientes buscan identificar variables psicológicas que influyen en la reactividad al estrés, ya que la literatura apoya la idea de que la percepción subjetiva del estrés está influenciada por variables disposicionales como la inteligencia emocional, sensibilidad a la ansiedad y la evitación experiencial, entre otras. Los resultados respaldan la necesidad de apuntar a cada una de estas variables disposicionales para reducir la reactividad al estrés emocional (Choi, Vickers, \& Tassone, 2014).

\section{Presente estudio}

Basado en lo antes expuesto los objetivos de este estudio son:

1) Estudiar si las diferentes etapas del desarrollo profesional de docentes hacen varíar los niveles de capital psicológico, engagement y burnout en docentes universitarios.

2) Estudiar la relación de las etapas del desarrollo profesional, del capital psicológico y el engagement laboral con el síndrome de burnout de los docentes universitarios.

\section{Método}

En el presente estudio es empírico utilizando metodología cuantitativa, el alcance del estudio es de tipo correlacional. El primer objetivo del estudio se pretende analizar por diferencia de grupos las variaciones del capital psicológico, el engagement docente y el burnout laboral según las etapas del desarrollo profesional de docentes universitarios. En el segundo objetivo se analiza la relación de las variables en estudio.

\section{Participantes y procedimiento}

Para efectuar el estudio, se conformó una muestra no probabilística y por conveniencia, integrada por 250 docentes universitarios argentinos. El 51\% de los participantes eran mujeres y el $49 \%$ hombres, con una media de edad de 39 años $(M=39.5$ y un DT $=11.15)$. El $40 \%$ de los 
participantes tiene título habilitante de grado y el $60 \%$ de los participantes cuenta con título de postgrado. El 38\% de los participantes ejercen la docencia universitaria hace 5 años o menos, el $27 \%$ lo hace entre 6 y 10 años y el 35\% hace más de 11 años que ejerce la docencia universitaria.

Para la recolección de los datos nos contactamos con las autoridades de tres universidades argentinas (una de gestión pública y dos de gestión privada) a quienes se les explicaron los objetivos de la investigación y se solicitó la información de contacto de sus docentes universitarios. Una vez obtenidos los permisos instituciones los docentes recibieron vía correo electrónico la invitación a participar del estudio. Se les explicó que su participación sería voluntaria, anónima y que la información no sería compartida con la institución académica. Aquellos docentes que accedieron participar completaron un consentimiento informado y se les otorgaron los protocolos con las escalas de evaluación. Los docentes universitarios no recibieron ningún tipo de recompensa por participar del estudio.

\section{Instrumentos}

Capital psicológico. Se utilizó la versión abreviada del Psychological Capital Questionnaire propuesta por Luthans, Avolio, Avey y Norman (2007), y validada en argentina por Omar, Salessi y Urteaga (2004). La escala incluye 12 ítems que evalúan las 4 dimensiones del capital psicológico: autoeficacia (ej. ítem "Me siento seguro presentando información a un grupo de colegas", Alfa de Cronbach para el estudio = .85, IC: .81, .88), esperanza (ej. ítem "Si me encuentro con un problema en el trabajo, puedo pensar muchas maneras de salir de él" Alfa de Cronbach para el estudio $=.83$, IC: .79, .86), resiliencia (ej. ítem "Puedo atravesar las situaciones difíciles en el trabajo porque he experimentado dificultades antes", Alfa de Cronbach para el estudio $=.71$, IC: .64, .75) y optimismo (ej. ítem "Soy optimista acerca de lo que me va a pasar en el futuro en lo que respecta al trabajo", Alfa de Cronbach para el estudio $=.83$, IC: $.78, .87$ ). Se les solicita a los docentes que evalúen cada afirmación utilizando una escala tipo Likert de 1 ("Muy en desacuerdo") a 6 ("Muy de acuerdo") puntos.

Engagement docente. Para evaluar el engagement laboral de los docentes universitarios se utilizó la versión corta de nueve ítems de la Utrecht Work Engagement Scale (UWES; Schaufeli \& Bakker, 2004) en su versión para argentina de Pujol-Cols, y Arraigada (2018). Dicha escala presenta niveles aceptables de validez y fue utilizada en varios estudios previos desarrollados en Argentina (ver Mesurado, Tortul, \& Schonfeld, 2018, Mesurado, Richaud, \& Mateo, 2016). La escala incluye elementos que miden tres dimensiones del engagement con el trabajo: vigor, dedicación y absorción. El vigor se caracteriza por invertir alta energía y fortaleza mental en el trabajo (ej. ítem "En mi trabajo me siento lleno de energía", Alfa de Cronbach para el estudio = .89, IC: .86, .91). Mientras que la dedicación se refiere a experimentar entusiasmo, inspiración, orgullo y desafío (ej. ítem "Cuando me levanto por las mañanas, tengo ganas de ir a trabajar", Alfa de Cronbach para el estudio $=.86$, IC: $.82, .88$ ). La absorción se refiere a estar concentrado e involucrado en el propio trabajo (ej. ítem "Estoy inmerso en mi trabajo", Alfa de Cronbach para el estudio $=.75$, IC: $.68, .81$ ). Se les pide a los participantes que utilicen una escala de 7 puntos dónde 1 significa Nunca y 7 Diariamente. La consistencia interna de la prueba total para este estudio fue Alfa de Cronbach = .91, IC: .90, .93.

Burnout laboral. Para evaluar el síndrome del burnout se utilizó el Oldenburg Burnout Inventory, desarrollado por Demerouti, Mostert y Bakker (2010) y adaptada a argentina en trabajos previos realizado por Mesurado \& Richaud (2017). La escala está compuesta por 16 ítems que evalúan dos aspectos del burnout: el agotamiento (ej. ítem "Hay días en los que me siento cansado antes de llegar al trabajo", Alfa de Cronbach para el estudio = .78, IC: .74, .82) y la falta de engagement (ej. ítems "Últimamente, me inclino a pensar menos en el trabajo y a hacerlo casi mecánicamente", Alfa de Cronbach para el estudio = .78, IC: .74, .82). La consistencia interna de la prueba total para este estudio fue Alfa de Cronbach $=.79$, IC: $.75, .82$. Se solicitó a los docentes que evalúen cada afirmación utilizando cuatro opciones de respuesta 1 ("Fuertemente de acuerdo") a 4 ("Fuertemente en desacuerdo") puntos. 
Experiencia docente. Se les consultó a los docentes acerca de la cantidad de años que ejercían la docente, luego las respuestas fueron codificadas en tres grupos Etapas 1, 2 y 3. Etapa 1 los participantes que ejercían la docencia universitaria hace 5 años o menos, el cual representó el $38 \%$ de los participantes. Etapa 2 los que ejercían la docencia universitaria entre 6 y 10 años, el cual representó el 27\% de los participantes. Por último, Etapa 3 aquellos que ejercían la docencia hace más de 11 años, los cuales representaron el 35\% de los participantes.

\section{Procedimiento estadístico}

Antes de realizar los análisis estadísticos para dar respuestas al primer objetivo del estudio se calculó la prueba de normalidad de Kolmogorov-Smirnov para cada variable en cada grupo de experiencia docente (etapa 1, 2 y 3). Los resultados indicaron normalidad en todas las dimensiones de todas las variables, a excepción de tres dimensiones de la variable capital psicológico (eficacia, resiliencia y optimismo) y dos dimensiones de engagement (dedicación y absorción). Esta falta de distribución normal en tres dimensiones de una variable capital psicológico se dio sólo en el grupo de los profesores que tenían más de 11 años de experiencia (etapa 3). Mientras que falta de distribución normal en dos dimensiones de la variable engagement se dio en los docentes entre 6 y 10 años de docencia (etapa 2). Por este motivo, para abordar el estudio del objetivo 1 se realizó análisis no paramétricos para estudiar las variables capital psicológico y engagement laboral. Mientras que para analizar la variable burnout se utilizó prueba paramétrica.

Para abordar el objetivo número 1 de la investigación se realizaron dos pruebas KruskalWallis en el primero se utilizó como VD las cuatro dimensiones del capital psicológico, en el segundo la tres dimensiones de engagement laboral. Por último, se realizó un análisis de variancia multivariado (MANOVA) para analizar el burnout. En todos los casos se utilizó como VI las tres etapas de experiencia docente (igual o menor a 5 años de experiencia, entre 6 y 10 años de experiencia y más de 11 años de experiencia docente).

Para responder al segundo objetivo del estudio se realizó un análisis de regresión por bloques dónde se incluyó como VI del bloque 1 las etapas de experiencia docente, en el bloque 2 se incluyeron las 4 dimensiones del capital psicológico y en el bloque 3 las 3 dimensiones del engagement laboral. Como VD se utilizó el nivel total de agotamiento laboral. Se utilizó esta prueba paramétrica luego de haber encontrado una distribución normal en las variables incluidas en el estudio utilizando Kolmogorov-Smirnov.

\section{Resultados}

\section{Capital psicológico, engagement y burnout laboral según las etapas de experiencia docente}

El primer objetivo de este estudio es analizar si varían los niveles de capital psicológico, engagement y burnout laboral en las diferentes etapas del desarrollo profesional de docentes universitarios. Para ello primero se calcularon los estadísticos descriptivos de media, desvío típico, asimetría y curtosis de cada una de las variables según el nivel de experiencia docente (Ver tabla 1). 
Tabla 1.

Estadística descriptiva de las variables incluidas en el estudio

\begin{tabular}{|c|c|c|c|c|c|c|c|c|c|}
\hline \multirow[b]{2}{*}{ Variables } & \multicolumn{3}{|c|}{$\begin{array}{c}\text { Etapa 1: } 5 \text { años o menos de } \\
\text { docencia } \\
\mathrm{n}=97\end{array}$} & \multicolumn{3}{|c|}{$\begin{array}{l}\text { Etapa 2: entre } 6 \text { y } 10 \text { años de } \\
\text { docencia } \\
n=65\end{array}$} & \multicolumn{3}{|c|}{$\begin{array}{c}\text { Etapa 3: } 11 \text { años o más de } \\
\text { docencia } \\
\mathrm{n}=88\end{array}$} \\
\hline & $\begin{array}{c}\mathrm{M} \\
(\mathrm{DT})\end{array}$ & Asimetría & Curtosis & $\begin{array}{c}\mathrm{M} \\
\text { (DT) }\end{array}$ & $\begin{array}{c}\text { Asime } \\
\text { tría }\end{array}$ & Curtosis & $\begin{array}{c}\mathrm{M} \\
(\mathrm{DT})\end{array}$ & $\begin{array}{c}\text { Asime } \\
\text { tría }\end{array}$ & Curtosis \\
\hline $\begin{array}{l}\text { Capital } \\
\text { psicológico }\end{array}$ & $\begin{array}{l}4.66 \\
(.93)\end{array}$ & -.96 & 1.25 & $\begin{array}{l}5.01 \\
(.82)\end{array}$ & -.60 & -.26 & $\begin{array}{l}5.04 \\
(.83)\end{array}$ & -1.9 & 6.58 \\
\hline Autoeficacia & & & & & & & & & \\
\hline Esperanza & $\begin{array}{l}4.58 \\
(.78)\end{array}$ & -.71 & .54 & $\begin{array}{l}4.66 \\
(.81) \\
\end{array}$ & -.48 & .47 & $\begin{array}{l}4.88 \\
(.70)\end{array}$ & 2.00 & 9.77 \\
\hline Resiliencia & $\begin{array}{l}4.33 \\
(.87)\end{array}$ & -.77 & .36 & $\begin{array}{l}4.69 \\
(.62)\end{array}$ & -.47 & .78 & $\begin{array}{l}4.74 \\
(.80)\end{array}$ & 1.15 & 2.99 \\
\hline Optimismo & $\begin{array}{c}4.51 \\
(1.08)\end{array}$ & -.94 & .72 & $\begin{array}{l}4.82 \\
(.95)\end{array}$ & -.91 & .93 & $\begin{array}{l}4.67 \\
(.93)\end{array}$ & 1.42 & 3.35 \\
\hline Engagement & & & & & & & & & \\
\hline Vigor & $\begin{array}{l}15.11 \\
(1.10)\end{array}$ & -.58 & .07 & $\begin{array}{c}5.13 \\
(1.21)\end{array}$ & -.92 & .24 & $\begin{array}{l}5.42 \\
(.89)\end{array}$ & -.45 & -.35 \\
\hline Dedicación & $\begin{array}{c}5.47 \\
(1.20) \\
\end{array}$ & -.97 & .81 & $\begin{array}{c}5.49 \\
(1.32)\end{array}$ & 1.40 & 2.40 & $\begin{array}{c}5.9 \\
(.82)\end{array}$ & -.66 & -.14 \\
\hline Absorción & $\begin{array}{l}5.38 \\
(.94)\end{array}$ & -.58 & .19 & $\begin{array}{c}5.32 \\
(1.13) \\
\end{array}$ & -1.11 & 2.13 & $\begin{array}{l}5.73 \\
(.90)\end{array}$ & -.53 & -.27 \\
\hline Burnout & & & & & & & & & \\
\hline Desconexión & $\begin{array}{l}22.00 \\
(.46) \\
\end{array}$ & .35 & .06 & $\begin{array}{l}2.01 \\
(.52)\end{array}$ & .47 & .22 & $\begin{array}{l}1.88 \\
(.43)\end{array}$ & -.03 & .04 \\
\hline Agotamiento & $\begin{array}{l}2.44 \\
(.37)\end{array}$ & .28 & .01 & $\begin{array}{l}2.35 \\
(.35)\end{array}$ & .68 & 1.3 & $\begin{array}{l}2.35 \\
(.38)\end{array}$ & .09 & 1.12 \\
\hline
\end{tabular}

Seguidamente se realizó una prueba de Kruskal-Wallis estudiando las diferencias en los niveles de capital psicológico de los docentes según las etapas del desarrollo profesional de docentes universitarios. Los resultados indican que existen diferencias estadísticamente significativas en los niveles de capital psicológico de acuerdo a la etapa de experiencia en nivel de eficacia percibida [Prueba Kruskal-Wallis $(2)=10.60, p \leq .01, \eta^{2}=.04$ ], nivel de resiliencia [Prueba Kruskal-Wallis $(2)=12.25, p \leq .01, \eta^{2}=.06$ ] y esperanza [ Prueba Kruskal-Wallis $(2)=$ $8.86, p \leq .01, \eta^{2}=.03$ ]. No se hallaron diferencias significativas en optimismo [ Prueba KruskalWallis $(2)=3.12, \mathrm{p}=.21]$. Posteriormente se analizaron las diferencias en las variables en estudio entre las diferentes etapas del desarrollo docentes utilizando la prueba U de Mann-Whitney. Los docentes que tenían 11 o más años de ejercicio de la profesión presentaron mayores niveles de autoeficacia [U de Mann-Whitney $=3160.5, p \leq 01$ ], esperanza [U de Mann-Whitney $=3223.5$, $p \leq 01]$ y resiliencia [U de Mann-Whitney $=3084.5, p \leq 01$ ] que los docentes que estaban transitando la primera etapa de desarrollo profesional (menos de 5 años de ejercicio). Así mismo, los docentes que tenían entre 6 y 10 años de ejercicio de la profesión presentaron mayores niveles de autoeficacia [U de Mann-Whitney $=2496.5, p \leq .05]$ y resiliencia [U de Mann-Whitney = $2459.0, p \leq 05]$ que los docentes de menos de 5 años de ejercicio.

Seguidamente una prueba de Kruskal-Wallis se utilizó para estudiar las diferencias en los niveles de engagement según las etapas del desarrollo profesional de docentes universitarios. Los resultados indican que existen diferencias estadísticamente significativas en los niveles de capital psicológico de acuerdo a la etapa de experiencia en nivel de absorción [Prueba Kruskal-Wallis $(2)=7.69, p \leq .05, \eta^{2}=.03$ ]. No se hallaron diferencias significativas en vigor [Prueba KruskalWallis $(2)=3.31, p=.19$ ] ni en dedicación [Prueba Kruskal-Wallis $(2)=5.76, p=.06$ ]. Luego se utilizó la prueba de U de Mann-Whitney para analizar la diferencia de los niveles de absorción entre los grupos. Los docentes que tenían 11 o más años de ejercicio de la profesión presentaron mayores niveles de absorción que los docentes que estaban transitando la primera etapa de desarrollo profesional (menos de 5 años de ejercicio) [U de Mann-Whitney $=3154.5, p \leq 01$ ] y la segunda etapa de desarrollo profesional (entre 6 y 10 años) [U de Mann-Whitney $=2270.0, p$ $\leq 05]$. 
Por último, se realizó un MANOVA en el que se analizó si varían los niveles de burnout en las diferentes etapas del desarrollo profesional de docentes universitarios. Los resultados indican que no hay diferencias estadísticamente significativas en el síndrome de burnout laboral [Taza de Hotelling $\left.F(4,490)=1.72, \mathrm{p}=.14, \eta^{2}=.01\right]$.

\section{Relación entre etapas del desarrollo docente, capital psicológico, y engagement laboral con el síndrome de agotamiento laboral.}

El segundo objetivo de este estudio es analizar la relación de las etapas del desarrollo profesional, el capital psicológico y el engagement laboral con el síndrome de burnout de los docentes universitarios. Para ello se llevó a cabo una regresión lineal por bloque. En el bloque 1, se introdujo la variable etapas del desarrollo docente, en el bloque 2, las 4 dimensiones del capital psicológico (autoeficacia, esperanza, resiliencia y optimismo), y en el bloque 3, las tres dimensiones de la variable engagement laboral (vigor, dedicación y absorción). Los tres bloques fueron utilizados como variables predictoras, mientras que el nivel total de agotamiento laboral o burnout fue utilizado como variable criterio.

Los resultados de los análisis indican que el modelo fue significativo en el bloque 1 [ $F$ $(1,248)=4.26, p \leq .05]$. El burnout estuvo significativamente relacionado por las etapas del desarrollo docente $(\beta=-.13$, IC: $-10,-.01)$. Los profesores con mayores niveles de experiencia presentaron menores niveles de burnout. El modelo basado en las etapas del desarrollo docente explicó el $2 \%$ de la variancia. En el segundo bloque también resultó significativo $[F(5,244)=$ $14.25, p \leq .001]$, la esperanza $(\beta=-.20$, IC: $-.17,-.02)$ y el optimismo $(\beta=-.24$, IC: $-.14,-.03)$ se encontraron relacionados con el burnout. Mayores niveles de esperanza y optimismo parecen desalentar la aparición del síndrome de agotamiento laboral. El segundo bloque explica el 21\% de la variancia. Por último, en el bloque 3 resultó significativo $[F(8,241)=25.28, p \leq .001]$, se identificó que las dimensiones de engagement laboral de vigor $(\beta=-.21$, IC: -.12, -.02) y dedicación ( $\beta=-.33$, IC: $-15,-.05)$ estuvieron relacionados también con la prevención del burnout. Este tercer bloque explicó el $23 \%$ de la variancia. Es decir, el modelo total explicó el $44 \%$ de las manifestaciones de burnout entre los docentes universitarios incluidos en este estudio. Todos los resultados de la regresión se muestran en la Tabla 2.

\section{Tabla 2.}

Resultados de la regresión por bloques es la predicción del burnout

Variables

$\mathrm{R}^{2} / \Delta \mathrm{R}^{2}$

Cambio en $\mathrm{F}$

$\beta$

(gl, gl error)

Bloque 1

$.02 / .02 *$

$\mathrm{F}(1,248)=4.26$

$-.13^{*}$

Etapas del desarrollo

docente

Bloque 2

$.23 / .21 * * *$

$F(4,244)=16.47$

$-.06$

Etapas del desarrollo

docente

Capital psicológico

Autoeficacia

Esperanza

Resiliencia 
Optimismo

Bloque 3

Etapas del desarrollo

docente

Capital psicológico

Autoeficacia

Esperanza

Resiliencia

Optimismo

$.44 / .23 * * *$

$F(3,241)=34.03$

$-.24 * *$

$-.01$

.01

Engagement

$-.21 * *$

Vigor

Dedicación

$-.33 * * *$

Absorción

$-.07$

Nota: ${ }^{*} p \leq .05 ; * * p \leq .01 ; * * * p \leq .001$

\section{Discusión}

El primer objetivo planteado en este estudio fue analizar si varían las características individuales de los docentes universitarios de acuerdo con el nivel de experiencia docente adquirida. Específicamente se analizó si los niveles de capital psicológico, engagement y burnout difieren en las diferentes etapas de experiencia profesional de docentes universitarios. Nuestros resultados indican que pueden hallarse diferencias estadísticamente significativas vinculadas al capital psicológico y al engagement. Los docentes con mayor nivel de experiencia (11 años o más) presentaban niveles más altos de eficacia, resiliencia y esperanza vinculados a su actividad docente que los profesores menos experimentados ( 5 años o menos de experiencia), así mismo los docentes que se encontraban en una etapa intermedia de experiencia docente (entre 6 y 11 años) presentaron mayores niveles de eficacia y resiliencia que los menos experimentados (5 años o menos de experiencia). Como era esperable, los docentes más experimentados presentaron mayores niveles de eficacia que los docentes que tienen menos años de experiencia, dado que con el paso del tiempo es lógico que crezcan en conocimiento y dominio de los contenidos que transmiten en sus clases, así como de habilidades pedagógicas para transmitir esos contenidos. Nuestros resultados van en la misma línea de investigaciones previas que identificaron altos niveles de motivación durante la formación inicial, mientras que la autoeficacia fue incrementándose a medida que avanzaban en la carrera docente (Hashmi \& Shaikh, 2011), así mismo otros estudios presentan que los docentes más experimentados tienen considerablemente mayores niveles de competencia que los menos experimentados (Evelein, Korthagen, \& Brekelmans, 2008).

Por otro lado, es probable que los docentes más experimentados hayan transitado por diferentes situaciones adversas en el campo laboral y personal, que los haya fortalecido a la vez que los hizo más resilientes para enfrentar las contradicciones laborales que los docentes menos experimentados. Es probable también que si el profesor experimentado alcanzó una dedicación apasionada a la actividad docente amplíe sus experiencias positivas en relación con su trabajo. Estas experiencias positivas, luego ayudan poderosamente a afrontar los conflictos. 
Además, el desarrollo de una actitud de esperanza frente a su trabajo puede llevar a los docentes experimentados a perseverar más en los objetivos hasta alcanzar situaciones de éxito y mantenerlas en el tiempo, que los docentes que recién comienzan su carrera académica. A su vez, la pasión orientada hacia una meta, mantiene y refuerza el compromiso y la acción (CardelleElawar \& Sanz, 2010; Marchesi \& Díaz Fouz, 2007).

Los resultados encontrados en este estudio indican que los niveles de engagement entre los docentes con diferentes niveles de experiencia, difieren sólo en la dimensión absorción. Los docentes más experimentados informan mayores niveles de absorción en su tarea docente que los menos experimentados. Es probable que la experiencia previa contribuya a su capacidad de concentración y absorción durante el trabajo debido al entrenamiento adquiriendo con los años. Sin embargo, no se hallaron diferencias en las otras dimensiones del engagement vigor y dedicación. Lo que indicaría que el trabajo docente tiene unas enormes posibilidades de generar una alta motivación intrínseca y un alto compromiso personal y profesional en las personas que lo desempeñan (González Torres, 2003) independientemente de los niveles de experiencia adquirida por el docente.

Por otro lado, nuestros estudios no identificaron diferencias en los niveles de burnout en relación con las etapas de experiencia laboral de los docentes universitarios. Tal vez, no hallamos diferencias porque los niveles de agotamiento laboral no varían de una etapa de experiencia docente a otra, sino que lo que puede cambiar son los tipos de estresores. Investigaciones previas encontraron que las fuentes de estrés cambian a lo largo de la carrera docente, así en el caso de los docentes más jóvenes fueron los alumnos y las condiciones laborales, mientras que en los más experimentados la fuente principal fue el entorno social (Flores Ramírez, 2002). De allí que coincidan estudios anteriores (Murillo Torrecilla, 2007; Veldman, van Tartwijk, Brekelmans, \& Wubbels, 2013), en la necesidad de elaborar políticas integradas que tiendan a formar docentes motivados y competentes que mantengan un nivel de engagement docente a lo largo de su carrera docente.

El segundo objetivo de nuestro trabajo fue analizar la relación de las etapas del desarrollo profesional, del capital psicológico y el engagement laboral con el síndrome de burnout en docentes universitarios. Nuestros resultados indican que la relación que tiene la experiencia docente en prevenir los niveles de burnout es muy baja (alrededor del 2\%). Sin embargo, las características individuales como el capital psicológico y los niveles de engagement se presentan como aspectos importantes en la prevención de este síndrome, explicando el $21 \%$ y el $23 \%$ de la variancia respectivamente. Específicamente encontramos que cuando los docentes informan altos niveles de esperanza y optimismo vinculados a su actividad docente esas características previenen la aparición de síndromes de agotamiento laboral. De allí que será importante fomentar en los docentes atribuciones positiva, basadas en la realidad objetiva, sobre sus logros educativos actuales y futuro. Lo cual los llevaría a perseverar en sus objetivos con la esperanza de alcanzar las metas propuestas. De estos resultados se deriva la necesidad de que las autoridades de las instituciones educativas sean capaces de estimular a los docentes universitarios en esa dirección, apoyarlos en su tarea y metas docentes, de tal modo que no se sientan solos frente al desafío de la educación del alumnado.

Por otro lado, es importante remarcar que el desarrollo de engagement laboral en los docentes también previene de la aparición de agotamiento laboral. Específicamente las dimensiones vigor (altos niveles de energía, perseverancia y esfuerzo en la labor) y dedicación (niveles elevados de implicación, entusiasmo, inspiración y reto en las tareas ocupacionales) están relacionadas con la inhibición del burnout. Es evidente que dedicarse a la educación con vigor y dedicación significa mucho más que tener un empleo. Al respecto, algunos autores hacen referencia que experimentar el trabajo docente como vocación, motiva y mantiene el empeño de los profesores (González Torres, 2003), de este modo puede prevenir el síndrome del burnout. El engagement que genera la docencia universitaria tal vez llena de sentido su actividad cotidiana lo que permite resignificar y atenuar los desafíos y el cansancio provocado por la tarea docente. 
A modo de conclusión, se puede decir que una de las principales contribuciones de este trabajo para la comunidad académica es el análisis de las variables en estudios (experiencia docente, capital psicológico, engagement y burnot) en un país específico de Latinomérica como Argentina en dónde los docentes Universitarios atraviesan fuertes presiones laborales. Estos hallazgos nos muestran que el síndrome del burnout, en esta población específica, no se debe principalmente al cansancio vinculado a los años de ejercicio docente, sino más bien a características individuales de los docentes, así como a la falta de engagement con la tarea.

\section{Limitaciones y futuros estudios}

Una de las limitaciones más importantes que presenta este estudio es que fue desarrollado utilizando un diseño de tipo transversal para la recolección de los datos; lo que no nos permite asegurar que las diferentes etapas de experiencia docente provoquen cambios en las características personales de los docentes universitarios. Así mismo, no nos permite analizar la trayectoria del capital psicológico, el engagement y el burnout a lo largo del tiempo ya que no hicimos un seguimiento de los mismos docentes en cada etapa de experiencia académica. Otro aspecto a tener en cuenta es que en este trabajo se operacionalizó la experiencia docente teniendo sólo en cuenta la antigüedad en años. En futuros estudios se podría enriquecer esta operacionalización integrando otros aspectos de la experiencia docente tales como el grado de estudios, el niveles o tipo de contratación que tengan los docentes con la institución, las horas frente a grupo, número de grupos, números de alumnos entre otros.

Por otro lado, el tipo de diseño utilizado nos habilita para analizar las relaciones entre la experiencia docente, el capital psicológico y el engagement con el burnout, pero no nos habilita para identificar estas variables como predictoras de esta sintomatología a la que pueden estar expuestos los docentes. Por último, otra limitación que encontramos y que podría tenerse en cuenta en futuros estudios es que la selección de los participantes fue de tipo intencional no probabilístico lo que también limita la generalización de los resultados a otros docentes universitarios.

\section{Referencias}

Bakker, A., Rodríguez-Muñoz, A., \& Derks, D. (2012). La emergencia de la psicología de la salud ocupacional positiva. Psicothema, 24(1), 66-72.

Botero Álvarez, C. C. (2012). Riesgo psicosocial intralaboral y "burnout" en docentes universitarios de algunos países latinoamericanos. Cuadernos de Administración, 28, 117132. https://doi.org/10.25100/cdea.v28i48.460

Cardelle-Elawar, M., \& Sanz, M. (2010). Looking at teacher identity through self-regulation. Psicothema, 22(2), 293-298.

Choi, K., Vickers, K., \& Tassone, A. (2014). Trait emotional intelligence, anxiety sensitivity, and experiential avoidance in stress reactivity and their improvement through psychological methods. Europe's Journal of Psychology, 10(2), 376-404. doi: https://doi.org/10.5964/ejop.v10i2.754

Darrigrande, J. L., \& Durán, K. (2012). Síndrome de burnout y sintomatología depresiva en profesores: relación entre tipo de docencia y género en establecimientos educacionales subvencionados de Santiago de Chile. Revista Iberoamericana sobre Calidad, Eficacia y Cambio en Educación, 10(3), 73-87.

De la Torre, M. J., \& Casanova, P. F. (2007). Comparative analysis of expectancies of efficacy in in-service and prospective teachers. Teaching and Teacher Education, 23 doi: https://doi.org/10.1016/j.tate.2007.02.005

Demerouti, E., \& Bakker, A. B. (2008). The Oldenburg Burnout Inventory: A good alternative to measure burnout and engagement. In J. R. B. Halbesleben (Ed.). Handbook of Stress and Burnout in Health Care (pp. 65-78). Nova Science Publishers Inc: New York, United States. 
Demerouti, E., Mostert, K., \& Bakker, A. B. (2010). Burnout and work engagement: a thorough investigation of the independency of both constructs. Journal of occupational health psychology, 15(3), 209-222. doi: https://doi.org/10.1037/a0019408

Dierendock, D., Buunk, B., \& Schaufeli, W. B. (2001a). Burnout and inequity among human service professionals: a longitudinal study. Journal of Occuparional Health Psychology, $6(1), 43-52$.

Evelein, F., Korthagen, F., \& Brekelmans, M. (2008). Fulfilment of the basic psychological needs of student teachers during their first teaching experiences Teaching \& Teacher Education, 24(5), 1137-1148. doi: https://doi.org/10.1016/j.tate.2007.09.001

Flores Ramírez, M. D. (2002). El factor humano en la docencia de educación secundaria: un estudio de la eficacia docente y el estrés a lo largo de la carrera profesional. (Tesis Doctoral), Universitat Autònoma de Barcelona, Barcelona. Recuperado de http://www.tdr.cesca.es/handle/10803/4734

González Torres, M. C. (2003). Claves para favorecer la motivación de los profesores ante los retos educativos actuales. ESE. Estudios sobre Educación, 5, 63-83.

Gutiérrez, M., Tomás, J. M., \& Alberola, S. (2018). Apoyo docente, compromiso académico y satisfacción del alumnado universitario. Estudios sobre Educación, 55, 535-555. doi: https://doi.org/10.15581/004.35.535-555

Hashmi, M. A., \& Shaikh, F. M. (2011). Comparative Analysis of the Effect of Teacher Education on Motivation, Commitment, and Self Efficacy. New Horizons (1992-4399), 10(2), 54-58.

Legé, L., López Pell, A. F., \& Fagnani, J. P. (2012). Evaluación del capital psíquico y valores en una institución universitaria. Revista Electrónica de Psicología Iztacala, 15(4), 1356-1397.

Lorente, L., \& Vera, M. (2010). Las organizaciones saludables: El engagement en el trabajo. Gestión Prácticas de Riesgos Laborales, 73, 16-30.

Lozano-Paz, C. R., \& Reyes-Bossio, M. (2017). Docentes universitarios: Una mirada desde la Autoeficacia general y engagement laboral. Revista Digital de Investigación en Docencia Universitaria, 11, 134-148. doi: http://dx.doi.org/10.19083/ridu.11.503

Luthans, F., Avolio, B., Avey, J., \& Norman, S. (2007). Positive psychological capital: measurement and relationship with performance and satisfaction. Personnel Psychology, 60(3), 541 - 572. doi: https://doi.org/10.1111/j.1744-6570.2007.00083.x

Luthans, F., Youssef, C., \& Avolio, B. (2007). Psychological capital: Developing the human competitive edge. New York: Oxford University Press.

Marchesi Ullastres, A., \& Díaz Fouz, T. (2007). Las emociones y los valores del profesorado. Madrid: Fundación Santa María.

Maslach, C., \& Jackson, S. E. (1981). The measurement of experienced burnout. Journal of Occupational Behavior, 2(99), 99-113. doi: https://doi.org/10.1002/job.4030020205

Mesurado, B., \& Richaud, M.C. (2017). Engagement and Burnout: the role of Personality Trait, Basic Psychological Needs and Academic Flow. Society for Research in Child Development (SRCD) Biennial Meeting. Lugar: Austin, USA.

Mesurado, B., Richaud, M.C, \& Mateo, J. N. (2016). Engagement, flow, self-efficacy, and eustress of university students: a cross-national comparison between the Philippines and Argentina. The Journal of psychology, 150(3), 281-299. doi: https://doi.org/10.1080/00223980.2015.1024595

Mesurado, B., Tortul, C. \& Schonfeld, F. (2018). Cinco grandes rasgos de personalidad: su relación con el flujo y el compromiso académico. Contextos de Educación, 20(25), 150158.

Murillo Torrecilla, J. C. (2007). Evaluación del desempeño y carrera profesional docente un estudio comparado entre 50 países de América y Europa. Chile: UNESCO.

Omar, A., Salessi, S., \& Urteaga, F. (2014). Diseño y validación de la escala CapPsi para medir capital psicológico. Liberabit, 20(2), 315-323.

Páramo, P. (2008). Factores psicosociales asociados a la evaluación del docente. Educación y Educadores, 11-30.

Ponce Gutiérrez, J. C., \& Yáber Oltra, G. (2012). Capital psicológico como predictor del comportamiento organizacional ciudadano en el ambiente universitario. Anales de la Universidad Metropolitana, 12(2), 167-185. 
Pujol-Cols, L. y Arraigada, M. (2018). Propiedades psicométricas de la versión corta de la Utrecht Work Engagement Scale en trabajadores argentinos. Pensamiento Psicológico, 16(2), 3145. doi: https://doi.org/10.11144/Javerianacali.PPSI16-2.ppvc

Salanova, M., Schaufeli, W. B., Llorens Gumbau, S., Peiró Silla, J. M., \& Grau Gumbau, R. M. (2000). Desde el burnout al engagement: ¿una nueva perspectiva? Revista de Psicología del Trabajo y de las Organizaciones, 16( 2), 117-134

Schaufeli, W., \& Bakker, A. B. (2004). Job demands, job resources, and their relationship with burnout and engagement: a multi-sample study. Journal of Organizational Behavior, 25, 293-315. doi: https://doi.org/10.1002/job.248

Seligman, M., \& Csikszentmihalyi, M. (2000). Positive Psychology: An Introduction. American Psychologist, 55(1), 5-14.

Silvero Miramón, M. (2007). Estrés y desmotivación docente: el síndrome del "profesor quemado" en educación secundaria. ESE. Estudios sobre Educación, 12, 115-138.

Siu, O., Bakker, A., \& Jiang, X. (2014). Psychological capital among university students: Relationships with study engagement and intrinsic motivation. Journal of Happiness Studies, 15, 979-994. doi: https://doi.org/10.1007/s10902-9459-2

Veldman, I., van Tartwijk, J., Brekelmans, M., \& Wubbels, T. (2013). Job satisfaction and teacher-student relationships across the teaching career: Four case studies. Teaching \& Teacher Education, 32, 55-65. doi: https://doi.org/10.1016/j.tate.2013.01.005 